\title{
EFFECT OF THE AVERAGE VELOCITY OF THE FREE PART OF THE SEMIFINISHED PRODUCT ON THE PROCESS OF PNEUMOTHERMAL FORMING IN THE SUPERPLASTIC REGIME
}

\author{
A. K. Shmakov, ${ }^{1}$ V. V. Mironenko, ${ }^{1}$ \\ K. K. Kirishina, ${ }^{1}$ A. S. Stanislavchik, ${ }^{1}$ \\ and V. V. Kotov 2,3
}

UDC 621.7 .04

Results are presented from two experiments involving the pneumothermal forming of a cellular product of alloy OT4-1 in the superplastic regime. It was determined that the average velocity of the free part of the semifinished product must be taken into account when using a model in which the stresses depend directly on the deformation rate.

Keywords: pneumothermal forming of disk-shaped parts, superplastic effect, temperature-speed conditions for deformation.

The process of pneumothermal forming in the superplastic regime cannot be carried out without strictly adhering to the parameters specified for the forming operation. Those parameters are determined by modeling the process and constructing a graph which describes the change in pressure over time with allowance for the constancy of the deformation rate. The graph is usually calculated and plotted by using a model of the material in which the stresses depend directly on deformation rate [1]:

$$
\sigma=K \dot{\varepsilon}^{m}
$$

where $K$ is a proportionality factor; $\dot{\varepsilon}$ is the deformation rate; $m$ is the strain-hardening modulus; and $\sigma$ is the stress.

The behavior of the material during pneumothermal forming in the superplastic regime is described fairly simply by this model, but many factors are not accounted for. We chose a cellular 1-mm-thick part of alloy OT4-1 (Fig. 1) to study the effect of one of those factors - the average velocity of the free part of the semifinished product.

The first stage of the investigation is the creation of a finite-element model of the semifinished product and the fixture. The model was constructed and the geometry of the elements in the forming operation (Fig. 2) was determined by using the CAD system Siemens NX.

The software package PAM-STAMP 2G, created by the French company ESI Group, was used as the CAE system for modeling pneumothermal forming in the superplastic regime. In accordance with the aforementioned model, material OT4-1 was assigned the following parameters to perform the modeling operation in this software:

- Young's modulus - $112 \mathrm{GPa}$;

\footnotetext{
${ }_{1}^{1}$ Irkutsk State Technical University, Irkutsk, Russia.

${ }^{2}$ ESI Group in Russia; e-mail: viacheslav.kotov@esi-group.com.

${ }^{3}$ Yeltsin Ural Federal University (UrFU), Yekaterinburg, Russia.
}

Translated from Metallurg, No. 1, pp. 24-26, January, 2013. Original article submitted December 25, 2012. 


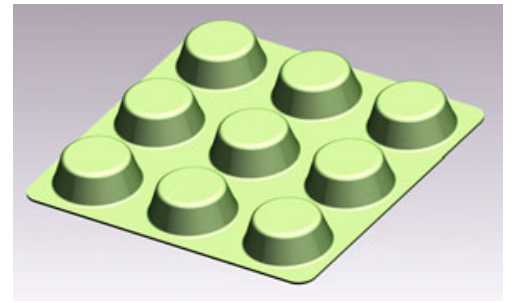

Fig. 1. The part being studied.

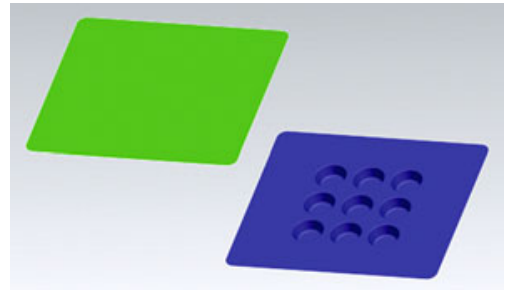

Fig. 2. Semifinished product and fixture for obtaining the part.

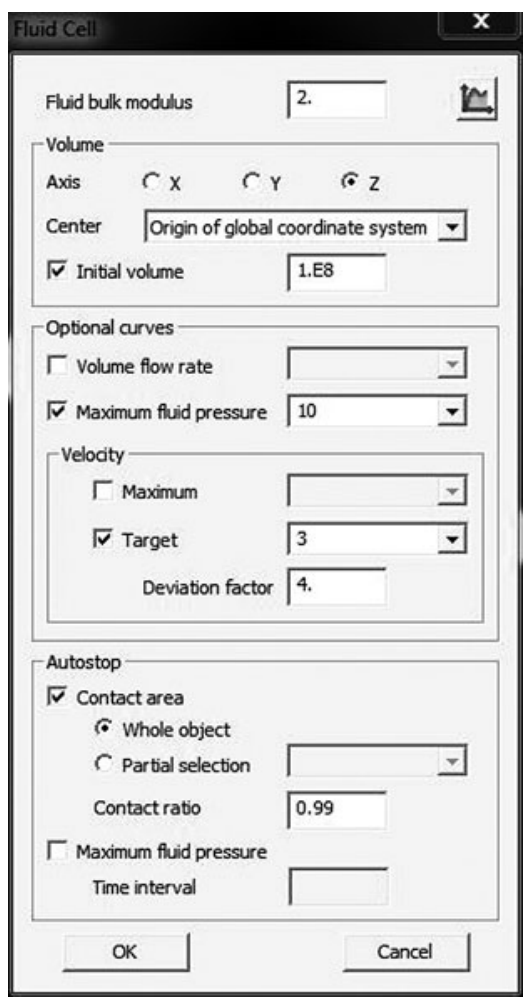

Fig. 3. Specifying the parameters of the liquid cell.

- Poisson's ratio - 0.333;

- density $-4.5 \cdot 10^{-6} \mathrm{~kg} / \mathrm{mm}^{3}$;

- proportionality factor $0.114116 \mathrm{GPa}$;

- deformation rate $-0.004 \mathrm{sec}^{-1}$ [2]; and

- strain-hardening modulus - 0.38 [2]. 


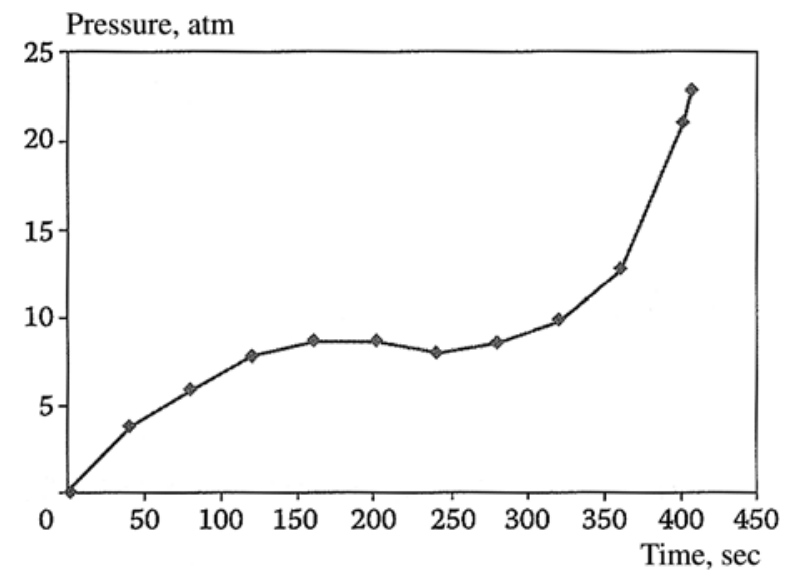

Fig. 4. Graph of the time change of pressure constructed from the results of the modeling.

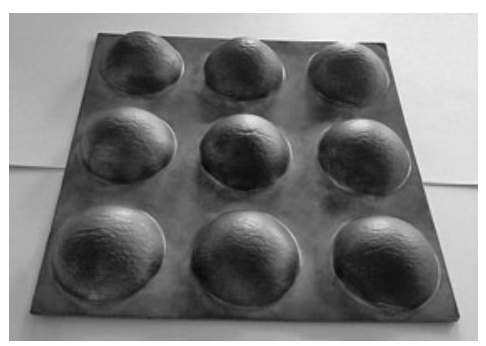

Fig. 5. The part obtained in an experiment performed in accordance with the graph (see Fig. 4).

The formation of the semifinished product solely by a reduction in its thickness is assigned as a boundary condition of the problem. This condition is satisfied by fixing the edges of the semifinished product, which ensures that it is deformed only as a result of a change in its thickness.

The liquid-cell method was used to load the semifinished product. In this method, the semifinished product undergoes deformation without discretization of the liquid medium. The following parameters were used to model this medium (Fig. 3):

- bulk deformation modulus - 2 (this parameter is used to calculate the change in pressure);

- initial volume of the liquid $-1 \cdot 10^{8} \mathrm{~mm}^{3}$;

- maximum pressure of the liquid - $10 \mathrm{GPa}$;

- average velocity of the free part of the semifinished product $-3 \mathrm{~mm} / \mathrm{sec}$; and

- value of the coefficient corresponding to termination of the calculation based on the degree of contact of the liquid with the semifinished product - 0.99 (i.e., the computation is ended when the degree of contact between the liquid and the semifinished product is $99 \%$ ).

Modeling the forming of the given part yielded the time dependence of the pressure. This dependence was used to ensure that the deformation rate remained constant. Here, the maximum pressure was $22.9 \mathrm{~atm}$ and the duration of the forming operation was $405 \mathrm{sec}$ (Fig. 4).

The parameters obtained from the modeling were used to perform an experiment. We subjected a sheet of titanium alloy OT4-1 to pneumothermal forming at $910^{\circ} \mathrm{C}$ in accordance with the graph of the change in pressure over time. When 


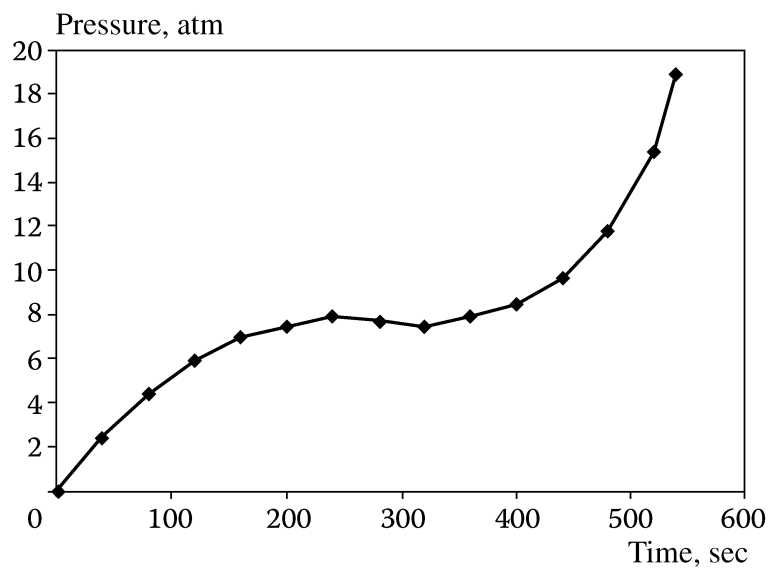

Fig. 6. Graph of the time change in pressure constructed from the results of modeling the process with a reduction in the average velocity of the free part of the semifinished product.

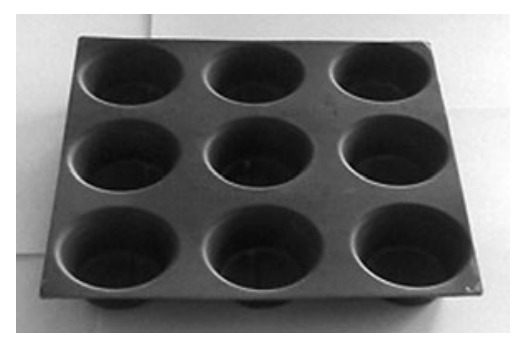

Fig. 7. The part obtained in an experiment conducted in accordance with another graph (see Fig. 6).

performed with the above-described parameters, the experiment failed to yield a part of the prescribed shape (Fig. 5) - the part "deviated from the regime."

The latter circumstance was evident from the large grain size in the deformation zone and the presence of two cracks on the part. The approach most commonly taken in this case is a reduction in deformation rate in the model and formation of the part over a longer period of time. However, we chose to use an alternative variant - we changed the average velocity of the free part of the semifinished product from $3 \mathrm{~mm} / \mathrm{sec}$ to $1 \mathrm{~mm} / \mathrm{sec}$. All the remaining data were left unchanged. As a result, we obtained a different graph to describe the change in pressure over time. The part is now formed over a longer period of time (Fig. 6).

In accordance with the result obtained from modeling the process in the second variant, the reduction in the velocity of the free part of the semifinished product led to a maximum pressure of $18.9 \mathrm{~atm}$ and a forming time of $538 \mathrm{sec}$. The graph was used to conduct a second experiment that employed the same material, which resulted in the formation of a part without defects (Fig. 7).

The most logical conclusion from these results is that the average velocity of the free part of the semifinished product affects the deformation rate in the model and, in the case being discussed, decreases it. Models were used for two variants of the processes to construct graphs that describe the dependence of the maximum deformation rate on time. An analysis of the graphs showed that the deformation rates were the same in the first and second cases and were constant (Fig. 8). 


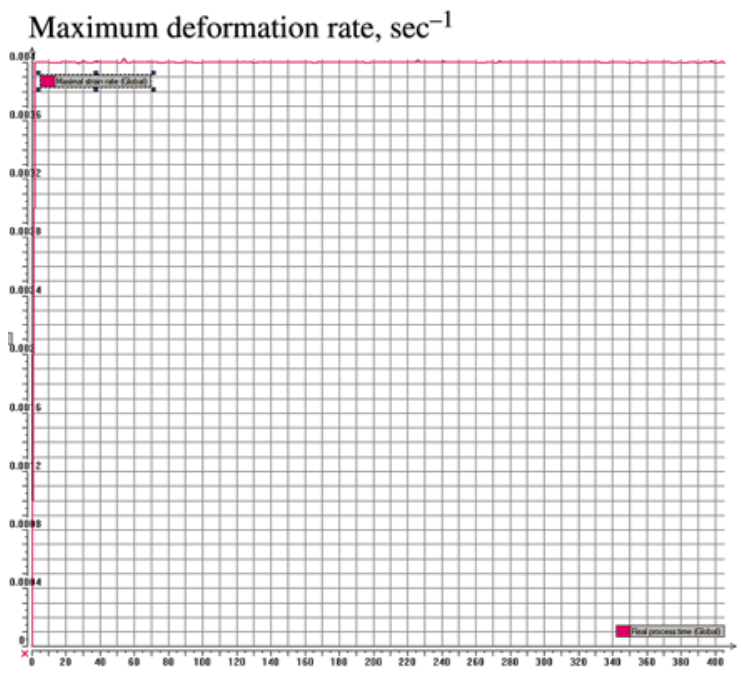

$a$

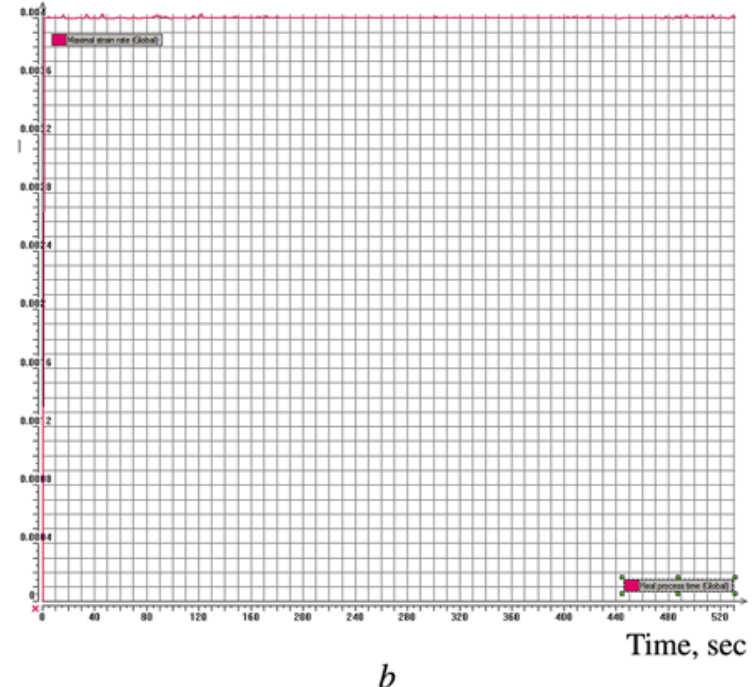

$b$

Fig. 8. Graphs of the time dependence of deformation rate in accordance with the first model $(a)$ and the second model $(b)$.

Conclusion. It is apparent from the models that have been developed and the completed experiments that it is important to consider a parameter such as the average velocity of the free part of the semifinished product when using the model in which the stress depends directly on the strain. Accounting for this parameter is important because when its value is optimum it ensures the formation of a quality part that has the parameters prescribed for it.

\section{REFERENCES}

1. E. N. Chumachenko, O. M. Smirnov, and M. A. Tsepin, Superplasticity: Materials, Theory, Technology [in Russian], Kom Kniga, Moscow (2009).

2. O. M. Smirnov, Metal-Shaping in the State of Superplasticity [in Russian], Mashinostroenie, Moscow (1979). 